\title{
Probabilistic seismic demand model for pounding risk assessment
}

\author{
E. Tubaldi ${ }^{1}$, F. Freddi ${ }^{2}$, M. Barbato ${ }^{3}$ \\ ${ }^{1}$ Department of Civil Engineering - Imperial College London, South Kensington Campus, London, SW7 2AZ, UK; \\ E-mail: e.tubaldi@ic.ac.uk \\ ${ }^{2}$ School of Engineering, University of Warwick, Coventry, CV4 7AL, UK; E-mail: F.Freddi@warwick.ac.uk \\ (corresponding author) \\ ${ }^{3}$ Department of Civil \& Environmental Engineering, Louisiana State University and A\&M College \\ 3418H Patrick F. Taylor Hall, Nicholson Extension, Baton Rouge, Louisiana 70803, USA; \\ E-mail: mbarbato@1su.edu
}

\begin{abstract}
Earthquake-induced pounding of adjacent structures can cause severe structural damage and advanced probabilistic approaches are needed to obtain a reliable estimate of the risk of impact. This study aims to develop an efficient and accurate probabilistic seismic demand model (PSDM) for pounding risk assessment between adjacent buildings, which is suitable for use within modern performance-based engineering frameworks.

In developing a PSDM, different choices can be made regarding the intensity measures (IMs) to be used, the record selection, the analysis technique applied for estimating the system response at increasing $I M$ levels, and the model to be employed for describing the response statistics given the $I M$. In the present paper, some of these choices are analyzed and evaluated first by performing an extensive parametric study for the adjacent buildings modeled as linear singledegree-of-freedom systems, and successively by considering more complex nonlinear multidegree-of-freedom building models. An efficient and accurate PSDM is defined using advanced intensity measures and a bilinear regression model for the response samples obtained by cloud analysis. The results of the study demonstrate that the proposed PSDM allows accurate estimates of the risk of pounding to be obtained while limiting the number of simulations required.
\end{abstract}

KEY WORDS: Pounding, Performance-Based Design, Probabilistic Seismic Demand Model, Intensity Measure.

\section{INTRODUCTION}

The seismic-induced pounding between adjacent buildings with inadequate separation distance is an undesirable event that can cause major damage and even structural collapse [1, 2]. This issue is particularly relevant for structures located in metropolitan areas, due to limited availability of land space.

In the last thirty years, extensive research was carried out to estimate both experimentally and numerically the effect of pounding between adjacent structures such as buildings and bridges. In most cases, the structural pounding phenomenon was shown to be detrimental rather than beneficial to the seismic performance of adjacent buildings, with its main effect consisting in an increase of the acceleration and drift demand at various story levels [3-7]. Although different techniques have been developed to minimize these effects (e.g., [9-10]), the simplest and most effective approach to mitigate seismic pounding is to provide enough clearance between the 
adjacent structures, though this solution may not always be feasible due to lack of space [11].

In order to control pounding risk, design codes prescribe a minimum separation distance between adjacent buildings and provide simplified numerical procedures and analytical rules for estimating its value under a given seismic hazard scenario [12-15]. However, these code procedures are characterized by unknown safety levels and, thus, do not permit to control explicitly the risk of pounding [16, 17] or the consequences of the impact [18]. In [16], a methodology was proposed to evaluate the risk of pounding between adjacent systems. This methodology was efficiently applied to the case of buildings modeled as linear systems, for which analytical techniques can be efficiently employed to estimate with good accuracy the response statistics under the uncertain earthquake input. Based on the results presented in [16], a reliability-based methodology was proposed in [17] for the design of the separation distance between adjacent buildings which corresponds to a target probability of pounding during the design life of the buildings. In [18], an alternative methodology has been proposed to design the separation distance between adjacent buildings by controlling also the consequences of the impact in term of increase of displacement demand for the systems.

Despite the advancement made by these works in the definition of the separation distance between adjacent buildings, further studies are required to make these methodologies applicable in an efficient way to more complex nonlinear building models, which require the use of computationally expensive numerical simulations to accurately simulate the structural response. The objective of this paper is to develop an efficient probabilistic seismic demand model (PSDM) [19-22] for pounding risk assessment consistent with modern performance-based design frameworks such as the Pacific Earthquake Engineering Research Center framework [23, 24]. A PSDM is the outcome of probabilistic seismic demand analysis (PSDA), and consists in the analytical representation of the relation between a seismic intensity measure $(I M)$ and a measure of the structural response of interest, i.e., an engineering demand parameter $(E D P)$. In this specific case, the EDP of interest is the peak relative displacement between the adjacent buildings at the most likely impact location. The PSDM can be used to estimate the seismic vulnerability and the mean annual frequency (MAF) of pounding between adjacent buildings via convolution with the hazard curve of the site.

In the development of a PSDM, different choices can be made regarding the $I M$ to be employed, the record selection, the technique used in PSDA to estimate the response statistics for different $I M$ levels, and the model describing the EDP statistics given the IM. In the present paper, some of these choices are discussed and evaluated by considering models of adjacent buildings with different degree of complexity.

First, the case of two adjacent buildings modeled as linear single-degree-of-freedom (SDOF) systems is considered. An extensive parametric study is performed by exploring a wide range of situations, as described by the identified non-dimensional characteristic parameters that control the system seismic behavior. Different $I M$ s are proposed for the problem at hand, whose choice is motivated mainly by efficiency criteria. The parametric study results are utilized to evaluate the efficiency and sufficiency of the proposed IMs employed in conjunction with a PSDM widely employed in the literature $[25,26]$ and involving the linear regression of the seismic demand variation with respect to the $I M$ in the log-log space.

Successively, a more realistic structural model of two adjacent buildings described as nonlinear hysteretic multi-degree-of-freedom (MDOF) systems is considered, with the aim of evaluating the effectiveness and accuracy of the IMs and PSDM introduced for the buildings described as SDOF systems. A bilinear (in the log-log space) PSDM is also proposed to achieve a better fit of the seismic median demand and dispersion over the entire range of seismic excitation levels. Finally, comparisons are made between the risk estimates obtained by using the linear and 
bilinear PSDMs and the corresponding estimates obtained via incremental dynamic analysis [27] in order to evaluate and compare the accuracy of the proposed regression models.

\section{DEVELOPMENT OF A PSDM FOR POUNDING RISK ASSESSMENT}

\subsection{Probabilistic seismic demand analysis}

The risk of pounding between two adjacent buildings $\mathrm{A}$ and $\mathrm{B}$, where $\mathrm{A}$ denotes the building providing the largest contribution to the displacement demand at the most likely impact location, can be expressed in terms of the MAF, $v_{E D P}(\xi)$, with which the peak relative displacement between the adjacent buildings at the most likely impact location, $u_{r e l}$ (EDP of interest in this problem), exceeds the separation distance $\xi[16]$. It is noteworthy that the most likely impact location can be obtained for any given pair of adjacent buildings by using the buildings' structural response statistics. However, for buildings that are regular in elevation and have a structural response dominated by the first mode of vibration (such as those considered in this study), the most likely impact location corresponds to the roof level of the lower of the two buildings. For the remainder of this study, the most likely impact location is assumed to coincide with the roof level of the lower of the two adjacent buildings. Based on the total probability theorem, $v_{E D P}(\xi)$ is expressed as:

$$
v_{E D P}(\xi)=\int_{i m} G_{E D P \mid I M}(\xi \mid i m) \cdot\left|\mathrm{d} v_{I M}(i m)\right|
$$

in which $G_{E D P \mid I M}(\xi \mid \mathrm{im})=$ complementary cumulative distribution function $(\mathrm{CCDF})$ of $E D P=$ $u_{r e l}$ conditional to $I M=i m$, and $v_{I M}(i m)=$ MAF of exceedance of a specific value $i m$. In this paper, upper case symbols indicate random variables and lower case symbols denote specific realizations of the corresponding random variable. It is noted here that, for linear elastic structures, building A usually corresponds to the building with higher natural period of vibration (i.e., the period corresponding to the first mode of vibration).

The probabilistic description of the seismic intensity measure $I M$ through the MAF $v_{I M}(\mathrm{im})$ is the task of probabilistic seismic hazard analysis. The description of $G_{E D P \mid I M}(\xi \mid \mathrm{im})$ is the task of PSDA, and returns the PSDM, which is the object of this study. In general, the computation of $G_{E D P \mid I M}(\xi \mid i m)$ involves performing a series of time-history dynamic analyses of the structural system under a set of ground-motion records with $I M$ levels spanning in the range of interest. Then, a regression analysis of the EDP samples on the corresponding $I M$ values is usually carried out to obtain a synthetic probabilistic description of the seismic demand given $I M=i m$ [26].

The two major issues in defining a PSDM for the problem considered in this study are related to the choice of (1) an appropriate (i.e., efficient and sufficient) $I M$, and (2) a regression model for the relation between the $E D P$ and the $I M$. It is noteworthy that these two problems are strictly related, because the appropriateness of an $I M$, as described in next section, is usually quantified based on the results of regression analysis, and thus depends on the regression model employed.

\subsection{Candidate IMs for pounding risk assessment}


The choice of an appropriate $I M$ is a critical issue in PSDA because it affects the computational cost and the accuracy of the estimates of $G_{E D P \mid I M}(\xi \mid i m)$ and, thus, of $v_{E D P}(\xi)$. Usually, the $I M$ is selected based on efficiency, sufficiency, and hazard computability criteria $[20,21,26]$. The term 'efficiency' is related to the dispersion of the seismic demand for a given $I M$ value. An efficient $I M$ results in a relatively small variability of $E D P$ conditional on $I M$, thereby reducing the number of time-history analyses that are necessary to estimate $G_{E D P \mid I M}(\xi \mid i m)$ with adequate confidence [28]. The term 'sufficiency' refers to the statistical independence of the EDP with respect to typical ground motion characteristics such as magnitude $(M)$ and source-to-site distance $(R)$. For example, if an $I M$ sufficient with respect to $M$ and $R$ is employed for PSDA, Eqn. (1) can be applied to estimate $G_{E D P \mid I M}(\xi \mid i m)$ without being affected by the values of $M$ and $R$ of the records employed for the non-linear dynamic analyses. The 'hazard computability' of an $I M$ refers to the availability of a hazard curve or attenuation law for that $I M$, or to the effort required to derive a seismic hazard model in terms of that $I M$. It is noteworthy that the optimal $I M$ in terms of efficiency and sufficiency is the EDP itself [26]. However, directly computing $v_{E D P}$ via probabilistic seismic hazard analysis would usually require the use of an impractically large number of time-consuming nonlinear dynamic analyses of the structural model subject to ground motions from an array of $M$ and $R$ values. Furthermore, this operation should be repeated for each different structure considered. Thus, the best $I M$ should be chosen among those for which hazard curves or attenuation laws are readily available or easy to compute. In this paper, a regression model is fitted to the results of PSDA. Thus, the efficiency of the proposed IMs is measured by the degree of scatter about the regression fit, whereas their sufficiency is measured by the extent to which the residuals of the regression are statistically independent of $M$ and $R$ [20, $26,29]$. In addition to this sufficiency measure, the 'relative sufficiency measure' (RSM) of an $I M$ over another is also evaluated, as in [30].

Based on the previous considerations, it is advantageous in terms of efficiency and sufficiency to select an $I M$ that is as close as possible to the EDP of interest. Modal combination rules such as the absolute sum (ABS), square root of the sum of the squares (SRSS), and double difference combination (DDC) rules can provide approximate estimates of the relative displacement response between two adjacent systems in function of their spectral displacement [31]. Since a hazard model is usually available for the spectral displacements, these rules can be employed to define efficient $I M$ s for pounding risk assessment.

The simplest $I M$ that naturally stems from the use of spectral displacements is:

$$
I M_{1}=\gamma_{\mathrm{A}} S_{d}\left(T_{\mathrm{A}}\right)
$$

where $S_{d}\left(T_{\mathrm{A}}\right)$ denotes the spectral displacement at the fundamental period $T_{\mathrm{A}}$ and $\gamma_{\mathrm{A}}$ denotes the fundamental mode participation factor of building A. In computing $\gamma_{\mathrm{A}}$, the modal shape is normalized to have a unit displacement at the pounding location. This intensity measure is roughly proportional to the spectral acceleration, which is widely employed in PSDA of buildings for its sufficiency and efficiency [28]. However, in the problem considered here, this $I M$ could be not appropriate due to the potentially relevant contribution of both buildings' displacements to the peak relative displacement.

A more advanced $I M$ can be defined as:

$$
I M_{2}=\gamma_{\mathrm{A}} S_{d}\left(T_{\mathrm{A}}\right) \sqrt{1+R_{B A}^{2}}=I M_{1} \sqrt{1+R_{B A}^{2}}
$$


where $R_{B A}=\left[\gamma_{\mathrm{B}} S_{d}\left(T_{\mathrm{B}}\right)\right] /\left[\gamma_{\mathrm{A}} S_{d}\left(T_{\mathrm{A}}\right)\right]$. This $I M$ is very similar to that proposed by [26] to reduce (when compared to using $I M_{1}$ ) the dispersion of buildings' inter-story drift demand by accounting also for the contribution of their second vibration mode. In the present study, $I M_{2}$ is proposed to account for the contribution to the peak relative displacement response of both systems and can be directly related to the SRSS rule for estimating the peak relative displacement.

An even more advanced $I M$ can be defined as:

$$
I M_{3}=\gamma_{A} S_{d}\left(T_{A}\right) \sqrt{1+R_{B A}^{2}-2 \rho_{B A} R_{B A}}=I M_{1} \sqrt{1+R_{B A}^{2}-2 \rho_{B A} R_{B A}}
$$

where $\rho_{B A}$ denotes the correlation factor between the two buildings' responses [31]. This last $I M$ can be directly related to the DDC rule for peak relative displacement evaluation, which is in general more accurate than the ABS and SRSS rules, especially for close fundamental vibration periods $[16,31]$. A hazard curve can be easily derived for each of the proposed IMs when an attenuation law for $S_{d}\left(T_{i}\right)(i=\mathrm{A}, \mathrm{B})$ is available.

It is noteworthy that building structures usually behave as MDOF hysteretic systems. Thus, evaluation of the separation distance between adjacent buildings should account for the contribution of the relevant vibration modes of each building, as well as for their nonlinear behavior. Although several approximate criteria for determining the peak relative displacement between adjacent buildings with nonlinear behavior have been proposed in the literature, none of these criteria appears to be very accurate under a wide range of conditions [32]. In addition, attenuation relations for inelastic spectral displacements are usually not available. Thus, IMs based on peak relative displacement estimates accounting for nonlinear behavior in a simplified way are not considered in this study. Furthermore, the use of vector valued IMs would open a full range of alternative model forms, combinatorial expansion of the problem considering $I M$ pairs, and practical challenges in implementation in a risk assessment analysis procedure. Thus, also the use of advanced vector-valued $I M S$ is considered out of the scope of the present study. On the other hand, the peak ground acceleration $(P G A)$, referred to hereinafter as $I M_{0}$, is considered in this work as a basic reference scalar $I M$, since it is employed in many studies for evaluating the pounding probability of buildings [16, 33].

\subsection{Regression models for pounding risk assessment}

\subsubsection{Linear regression model}

PSDM are often built by using the following expression as regression model between EDP and a scalar $I M[25]$ :

$$
\ln E D P|I M=\ln a+b \ln I M+\ln \varepsilon| I M
$$

where the parameters $a$ and $b$, as well as the error variable $\varepsilon \mid I M$ need to be estimated via regression analysis in the log-log space of the EDPs samples given $I M$. The functional form given by Eqn. (5) is based on extensive regression analysis of the seismic response of steel structures [25]. The variable $\varepsilon \mid I M$ is assumed to be lognormally distributed, i.e., $\ln \varepsilon \mid I M$ follows a normal distribution with zero mean value and standard deviation $\beta_{\ln \varepsilon \mid M}(\mathrm{im})$. Thus, 
also the considered EDP follows a lognormal distribution and $\ln E D P \mid I M$ is normally distributed with mean value $\ln a+b \cdot \ln i m$ and standard deviation $\beta_{\ln E D P \mid I M}(\mathrm{im})$.

The assumed regression model permits to evaluate in closed form the CCDF $G_{E D P \mid I M}(\xi \mid i m)$ used in Eqn. (1) as [25]:

$$
G_{E D P \mid I M}(\xi \mid i m)=P[E D P|I M \geq \xi| i m]=\Phi\left(\frac{\ln a+b \cdot \ln i m-\ln \xi}{\beta_{\ln E D P \mid I M}(i m)}\right)
$$

where $\Phi(\cdot)$ denotes the standard normal cumulative distribution function. The plot of $G_{E D P \mid I M}(\xi \mid \mathrm{im})$ as a function of $I M$ is commonly denoted as fragility curve in the literature [34].

Different techniques can be used to generate the EDPs samples given $I M[22,25,28,35]$. In this study, cloud analysis is employed. The use of this technique is usually coupled with the assumption of homoscedasticity of the demand, i.e., the standard deviation of the EDP is assumed constant with respect to $I M$ as $\beta_{\ln E D P \mid I M}(\mathrm{im})=\beta$ [22].

It is noteworthy that, in the case of linear elastic behavior of the two adjacent systems, $b$ can be assumed equal to one, and the PSDM requires a simpler one parameter log-log linear regression. Other studies suggest to take $b=1$ even for PSDA of systems behaving nonlinearly, e.g., for estimating the maximum inter-story drift in building frames for some advanced $I M \mathrm{~s}$ [26]. Under these assumptions, the assessment of the efficiency and sufficiency of the $I M$ is greatly simplified [26].

Finally, it is observed that in developing a PSDM specific for pounding risk assessment, particular attention should be given to the demand samples to be considered. In fact, the buildings may collapse under the action of earthquakes characterized by high $I M$ levels (i.e., for high building displacement demands) independently from the occurrence of pounding. The EDPs samples corresponding to these earthquakes should be discarded in the regression analysis.

\subsubsection{Bilinear model}

It has been shown that the period elongation and hysteretic damping following buildings' yielding very often can result in smaller values of the peak relative displacement for buildings with nonlinear behavior than for those with linear behavior $[32,36]$. Thus, a linear relationship in the log-log plane between the $I M$ and the median response could be not valid for the entire $I M$ range of interest. Another situation in which a linear PSDM model can fail in properly describing the seismic demand is when two adjacent systems have the same behavior in the elastic range, but different yield displacements and nonlinear behaviors [37]. For low seismic excitation levels (under which the systems behave linearly), the relative displacement demand is zero. However, for seismic intensities under which the weaker system yields, the relative displacement demand can increase significantly for increasing $I M$ levels. The nonlinear building behavior is also expected to induce an increased dispersion of the EDPs values, due to the reduced efficiency of an $I M$ that is based on the elastic system properties. Thus, also the assumption of homoscedasticity could be not satisfied.

Several alternative techniques exist to solve these issues in accurately describing the EDP seismic demand [19, 21]. In this paper, a bilinear PSDM (Figure 1) is considered because of its simplicity and the small number of parameters involved in the fitting. The bilinear regression model has been employed in other PSDA studies, e.g., to describe the relationship between drift 
demand and seismic intensity in multi-story frame structures [38].

This bilinear regression model is described by the following expression:

$$
\ln E D P\left|I M=\left(a_{1}+b_{1} \ln I M\right) H_{1}+\left(a_{2}+b_{2} \ln I M\right)\left(1-H_{1}\right)+\ln \varepsilon\right| I M
$$

in which $a_{i}$ and $b_{i}(i=1,2)$ control the intercepts and the slopes of the $i$-th segment, respectively (see Figure 1), and $H_{1}$ denotes the step function (i.e., $H_{1}=1$ for $I M \leq I M^{*}$, and $H_{1}=0$ for $I M>I M^{*}$, where the parameter $I M^{*}$ identifies the breakpoint, which is defined as the point of intersection of the two segments, corresponding on average to the yielding of any of the two buildings). The value of $I M^{*}$ is obtained by solving the following equation:

$$
a_{1}+b_{1} \ln I M^{*}=a_{2}+b_{2} \ln I M^{*}
$$

By substituting Eqn.(8) into Eqn.(7), the following alternative expression is obtained:

$$
\ln E D P\left|I M=\left(a_{1}+b_{1} \ln I M\right) H_{1}+\left[a_{1}+\left(b_{1}-b_{2}\right) \ln I M^{*}+b_{2} \ln I M\right]\left(1-H_{1}\right)+\ln \varepsilon\right| I M
$$

In the problem considered in this paper, the breakpoint $I M^{*}$ is not known, and the model parameters $a_{1}, b_{1}, b_{2}, \ln I M^{*}$ can be estimated by performing ordinary nonlinear least square regression. The value $b_{1}=1$ can be assumed for the first segment describing the buildings' linear response. It is noteworthy that the use of a bilinear model permits to consider two different dispersions for the linear (first segment) and nonlinear (second segment) range of behavior, i.e., it allows to relax the assumption of homoscedasticity.

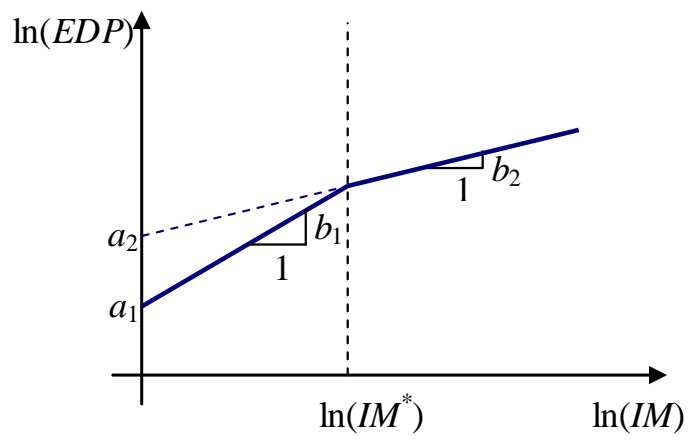

Figure 1. Illustration of bilinear regression model parameters.

\section{PARAMETRIC STUDY FOR ADJACENT BUILDINGS MODELED AS LINEAR SDOF SYSTEMS}

In this section, the adjacent buildings are modeled as linear elastic SDOF systems. An extensive parametric study for a wide range of system parameters is carried out to evaluate the accuracy and efficiency of the proposed $I M \mathrm{~s}$ in conjunction with the linear regression model for estimating the relative displacement demand. It is noteworthy that the use of linear elastic SDOF models for the buildings permits to reduce the number of parameters to be analyzed and varied in the parametric study and, thus, to explore wide ranges of situations while limiting the computational efforts. Furthermore, the use of linear structural models can be representative of many situations in which the buildings are very close one to each other and, thus, collide while 
vibrating in their linear range of behavior.

A dimensional analysis of the problem [39] reveals that, using an $I M$ whose dimension is a length, the normalized relative displacement response between two buildings undergoing freevibrations can be expressed as:

$$
\frac{u_{r e l}}{I M_{i}}=f\left(\frac{T_{\mathrm{A}}}{T_{\mathrm{B}}}, \zeta_{\mathrm{A}}, \frac{\zeta_{\mathrm{B}}}{\zeta_{\mathrm{A}}}\right) ; \quad i=1,2,3
$$

where $\zeta_{i}(i=\mathrm{A}, \mathrm{B})$ denotes the damping ratio corresponding to the first mode of vibration of each building.

By contrast, under seismic excitation, the peak relative displacement depends also on the frequency content and duration of the earthquake input. These effects for a given $I M$ are related to the natural vibration periods of the two buildings taken individually as:

$$
\frac{u_{r e l}}{I M_{i}}=f\left(T_{\mathrm{A}}, \frac{T_{\mathrm{A}}}{T_{\mathrm{B}}}, \zeta_{\mathrm{A}}, \frac{\zeta_{\mathrm{B}}}{\zeta_{\mathrm{A}}}\right) ; \quad i=1,2,3
$$

To reduce the number of parameters of the analysis, it is assumed here that $\zeta_{\mathrm{A}}=\zeta_{\mathrm{B}}=2 \%$. The vibration period $T_{\mathrm{A}}$ of building $\mathrm{A}$ is varied in the range $0-4 \mathrm{~s}$ (with an interval of $0.2 \mathrm{~s}$ ), whereas the ratio $T_{\mathrm{B}} / T_{\mathrm{A}}$ is varied in the range $0-1$ (with an interval of 0.1 up to 0.9 and of 0.025 from 0.9 to 1). The results corresponding to $T_{\mathrm{B}} / T_{\mathrm{A}}=1$ are obtained at the limit for $T_{\mathrm{B}} / T_{\mathrm{A}} \rightarrow 1$ from below, because $I M_{3}=0$ for $T_{\mathrm{B}} / T_{\mathrm{A}}=1$.

A set of $N_{g m}=240$ records taken from [40] is selected to account for the variability of the frequency content and duration of the seismic input. The set of records used in this study was obtained by excluding pulse-like records from the original set presented in [40]. Dynamic timehistory analyses are carried out under the selected records and the results are fitted by using a one-parameter linear regression model obtained by assuming $b=1$ in Eqn. (5). The parameter $a_{i}$ for the $i$-th $I M(i=1,2,3)$ is estimated as the $50^{\text {th }}$ percentile of the samples of the normalized demand $u_{r e l} / I M_{i}$, whereas the lognormal standard deviation (also called dispersion) $\beta_{i}$ is evaluated as [26]:

$$
\beta_{i}=\sqrt{\frac{\sum_{j=1}^{N_{g m}}\left[\ln \left(u_{r e l} / I M_{i}\right)_{j}-\ln \left(a_{i}\right)\right]^{2}}{N_{g m}-2}} ; \quad i=1,2,3
$$

Figure 2 reports the normalized median response $a_{i}$ as a function of $T_{\mathrm{A}}$ and $T_{\mathrm{B}} / T_{\mathrm{A}}$, for the different $I M$ s considered. In Figure 2(a), which shows the results obtained by using $I M_{0}=P G A$, the displacement is normalized as $u_{r e l} \omega_{\mathrm{A}}^{2} / I M_{0}$, where $\omega_{\mathrm{A}}=2 \pi / T_{\mathrm{A}}$ denotes the natural circular frequency of building $\mathrm{A}$, since the dimension of $P G A$ is of a length divided by a squared time. The relative displacement demand normalized to the $P G A$ exhibits a significant dependence on both $T_{\mathrm{A}}$ and $T_{\mathrm{B}} / T_{\mathrm{A}}$. It is observed that, for $T_{\mathrm{B}} / T_{\mathrm{A}}=0$, the values of $a_{0}$ shown in Figure 2(a) coincide with the median pseudo spectral accelerations of the records for the vibration period $T_{\mathrm{A}}$, normalized by the $P G A$.

For the $I M \mathrm{~s}$ based on spectral displacements (i.e., $I M_{1}, I M_{2}$, and $I M_{3}$ ), the values of the 
normalized relative displacement demand $a_{i}(i=1,2,3)$ are only slightly affected by the vibration period $T_{\mathrm{A}}$ of building $\mathrm{A}$. They slowly increase when $T_{\mathrm{B}} / T_{\mathrm{A}}$ increases from 0 to approximately 0.8 and decrease when $T_{\mathrm{B}} / T_{\mathrm{A}}$ increases from 0.8 to 1 . For $T_{\mathrm{B}} / T_{\mathrm{A}}=0 \div 0.8$ and $T_{\mathrm{A}} \geq 0.3 \mathrm{~s}$, the results carried out by using $I M_{2}$ and $I M_{3}$ are only slightly biased in estimating $a_{i}$ (i.e., $a_{i}$ assumes values close to one for $i=2,3$ ), whereas those evaluated by using $I M_{1}$ are more biased, because the contribution of system B to the relative displacement response is disregarded. In the same period ranges, $I M_{2}$ practically coincides with $I M_{3}$, because the correlation factor $\rho$ is almost zero for distant vibration periods. As $T_{\mathrm{B}} / T_{\mathrm{A}}$ approaches zero (from above), the normalized relative displacements $a_{i}(i=1,2,3)$ tend to slightly less than one. This phenomenon is due to the fact that the relative displacement tends to the displacement of building A, while $I M_{i}(i=1,2$, 3) approaches the peak absolute displacement of building A.

For $T_{\mathrm{B}} / T_{\mathrm{A}}$ approaching one (from below), the normalized relative displacement response tends to zero if $I M_{1}$ or $I M_{2}$ are employed, because the two systems vibrate in phase. $I M_{3}$ is less biased in estimating the peak displacement, because it accounts for the correlation between the adjacent buildings' responses. For $T_{\mathrm{B}} / T_{\mathrm{A}}$ approaching one, $I M_{3}$ tends to zero. However, $a_{3}$ tends to a finite value which depends on the system and ground motion properties (in fact, the DDC rule and thus $I M_{3}$ provide exact estimates of the peak relative displacement only in the case of stationary response to stationary white noise excitation).
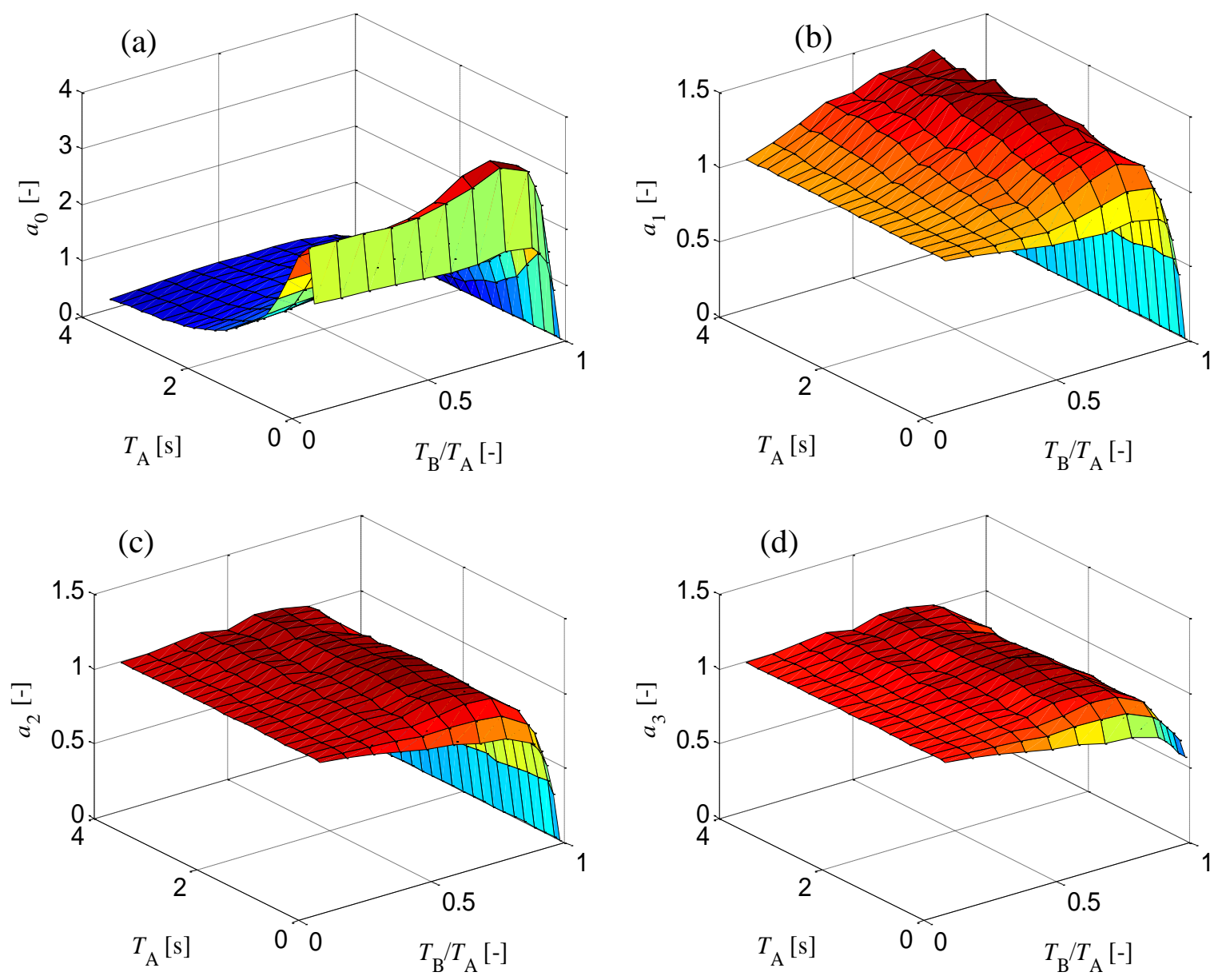

Figure 2. Normalized median relative displacements for different system vibration periods using as $I M$ : (a) $I M_{0}=P G A$, (b) $I M_{1}$, (c) $I M_{2}$, and (d) $I M_{3}$. 
Figure 3 reports the dispersions $\beta_{i}$ as a function of $T_{\mathrm{A}}$ and the ratio $T_{\mathrm{B}} / T_{\mathrm{A}}$, for the different $I M \mathrm{~s}$ considered. In general, the dispersion $\beta_{0}$ for $I M=P G A$ is very high, with values varying from about 0.50 to 1.20 (Figure 3(a)). As expected, $\beta_{i}$ is significantly lower for $I M$ s based on spectral displacements (Figure 3(b) through (d)). For $T_{\mathrm{B}} / T_{\mathrm{A}}$ in the range between 0 and $0.8, \beta_{1}$ assumes values lower than 0.30 , while $\beta_{2}$ and $\beta_{3}$ assume values lower than 0.20 . The higher efficiency of $I M_{2}$ and $I M_{3}$ is due to the fact that they account for the contribution of building $\mathrm{B}$ to the relative displacement demand. For $T_{\mathrm{B}} / T_{\mathrm{A}}$ approaching one (from below), $\beta_{i}$ increases significantly for $i=$ $1,2,3$, and $I M_{3}$ has an efficiency similar to that of $I M_{2}$. However, the values assumed by $\beta_{i}(i=1$, 2,3 ) remain lower than 0.40 in all cases considered here.
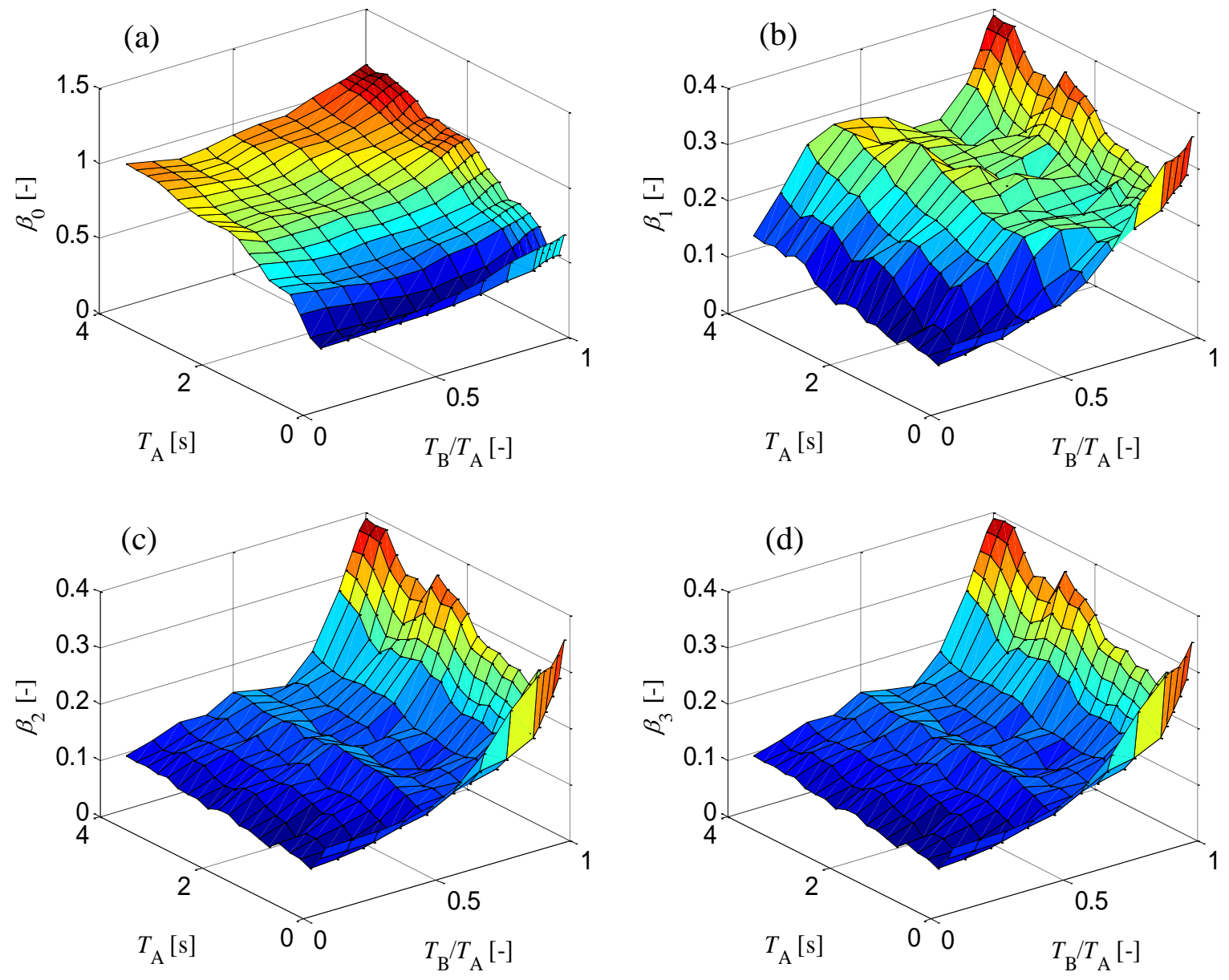

Figure 3. Relative displacement response dispersion $\beta$ for different system vibration periods using as $I M$ : (a) $P G A$, (b) $I M_{1}$, (c) $I M_{2}$, and (d) $I M_{3}$.

The sufficiency of the different $I M \mathrm{~s}$ with respect to $M$ and $R$ is also analyzed, for each combination of the parameters considered, by performing a multiple linear regression of the residuals $\left(\ln \varepsilon_{k} \mid I M\right.$ for $k=1,2, \ldots, N_{g m}$ ) jointly with respect to $M$ and $\ln (R)$ [26], and by evaluating the corresponding $\mathrm{p}$-values. These $\mathrm{p}$-values denote the probability of finding estimates of the coefficients of the regression multiplying $M$ and $\ln (R)$ at least as large (in absolute value) as those observed if, in fact, the true value were 0 . It is noteworthy that p-values lower than 0.05 suggest that the $I M$ is insufficient. Figure 4 reports the p-values corresponding to all the cases analyzed in the parametric study and obtained by considering $I M_{i}(i=0,1,2,3)$ as $I M$ (Figure 4(a) through (d), respectively). While $I M_{0}$ is largely insufficient, $I M_{2}$ is sufficient for a wide 
range of system properties. The other results of the sufficiency study demonstrate that $I M_{1}$ is more sufficient than $I M_{0}$ but less sufficient than $I M_{2}$, whereas $I M_{2}$ gives results comparable to $I M_{3}$ in terms of sufficiency. It is noteworthy that, in a few cases, even $I M_{2}$ and $I M_{3}$ are not sufficient.
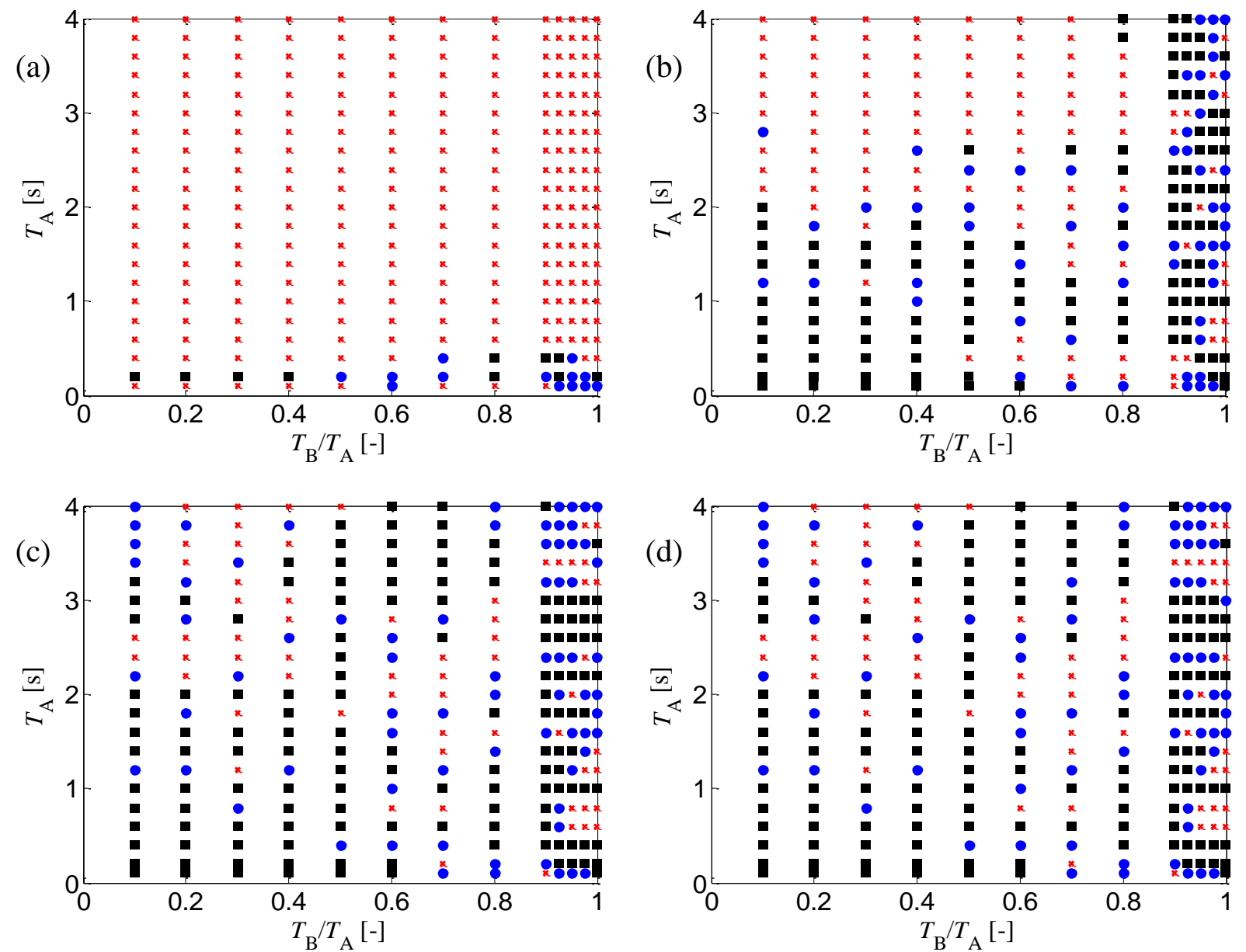

Figure 4. Variation with $T_{\mathrm{A}}$ and $T_{\mathrm{B}} / T_{\mathrm{A}}$ of the p-values for different system vibration periods using as $I M$ :

(a) $I M_{0}$; (b) $I M_{1}$; (c) $I M_{2}$; (d) $I M_{3}$. p-values ranges: ' $\times$ ' for p-values $<0.05$; ' $\bullet$ ' for $0.05 \leq$ p-values $<0.20$; - for pvalues $\geq 0.20$.

In order to provide a further comparison between the sufficiency properties of the $I M \mathrm{~s}$, the 'relative sufficiency measure' (RSM) of an IM over another is also evaluated by following the approach proposed in [30]. This measure quantifies the relative change of information regarding the $E D P$ of interest obtained by using two different $I M \mathrm{~s}$. The RSM of $I M_{i}$ over $I M_{j}$ for $i, j=0,1$, 2, 3 is calculated as:

$$
I\left(u_{r e l}\left|I M_{i}\right| I M_{j}\right)=\frac{1}{N_{g m}} \sum_{k=1}^{N_{g m}} \log _{2} \frac{p_{a_{i} \mid I M_{i}}\left(a_{i_{k}} \mid I M_{i}\right)}{p_{a_{j} \mid I M_{j}}\left(a_{j_{k}} \mid I M_{j}\right)}
$$

where $p_{E D P \mid I M}(E D P \mid I M)$ denotes the probability distribution function (PDF) (usually assumed to follow a lognormal distribution) of the EDP conditioned to the $I M$. A positive value of 
$I\left(u_{r e l}\left|I M_{i}\right| I M_{j}\right)$ means that, on average, $I M_{i}$ provides more information about the monitored $E D P$ than $I M_{j}$ and, hence, that $I M_{i}$ is more sufficient than $I M_{j}$.

Figure 5(a) and (b) show the improvements in terms of sufficiency obtained by using $I M_{1}$ instead of $I M_{0}$ and $I M_{2}$ instead of $I M_{1}$, respectively. In the majority of the cases, both $I\left(u_{\text {rel }}\left|I M_{1}\right| I M_{0}\right)$ and $I\left(u_{r e l}\left|I M_{2}\right| I M_{1}\right)$ assume positive values for the considered range of parameters investigated. Thus, in general, $I M_{1}$ is more sufficient than $I M_{0}$ and $I M_{2}$ is more sufficient that $I M_{1}$. The RSM $I\left(u_{\text {rel }}\left|I M_{1}\right| I M_{0}\right)$ assumes large positive values for low values of $T_{\mathrm{A}}$, decreases for increasing values of $T_{\mathrm{A}}$, assumes negatives values (in general of small absolute value) for some of the cases corresponding to $T_{\mathrm{A}} \geq 2.6 \mathrm{~s}$, and is only weakly sensitive to the ratio $T_{\mathrm{B}} / T_{\mathrm{A}}$. The RSM I $\left(u_{\text {rel }}\left|I M_{2}\right| I M_{1}\right)$ assumes positive values for all cases considered here, generally decreases for decreasing values of $T_{\mathrm{A}}$, and for a given $T_{\mathrm{A}}$ achieves a maximum value for $T_{\mathrm{B}} / T_{\mathrm{A}}$ close to 0.5 . For $T_{\mathrm{B}} / T_{\mathrm{A}}$ approaching $1, I\left(u_{\text {rel }}\left|I M_{2}\right| I M_{1}\right)$ tends to 0.5 for all values of $T_{\mathrm{A}}$. This result is due to the fact that, for $T_{\mathrm{B}} / T_{\mathrm{A}} \rightarrow 1, \quad I M_{2} \rightarrow \sqrt{2} \cdot I M_{1}$ and $p_{a_{2} \mid I M_{2}}\left(a_{2} \mid I M_{2}\right) \rightarrow \sqrt{2} \cdot p_{a_{1} \mid I M_{1}}\left(a_{1} \mid I M_{1}\right)$. The results for $I\left(u_{r e l}\left|I M_{2}\right| I M_{0}\right), I\left(u_{r e l}\left|I M_{3}\right| I M_{2}\right)$, $I\left(u_{r e l}\left|I M_{3}\right| I M_{1}\right)$, and $I\left(u_{r e l}\left|I M_{3}\right| I M_{0}\right)$ are not plotted here due to space constraint. However, they indicate that using $I M_{3}$ does not provide any significant advantage in terms of sufficiency when compared to using $I M_{2}$, and that $I M_{2}$ is always more sufficient than $I M_{0}$ with the exception of the cases corresponding to $T_{\mathrm{A}} \geq 3.8 \mathrm{~s}$ and $T_{\mathrm{B}} / T_{\mathrm{A}} \geq 0.95$.
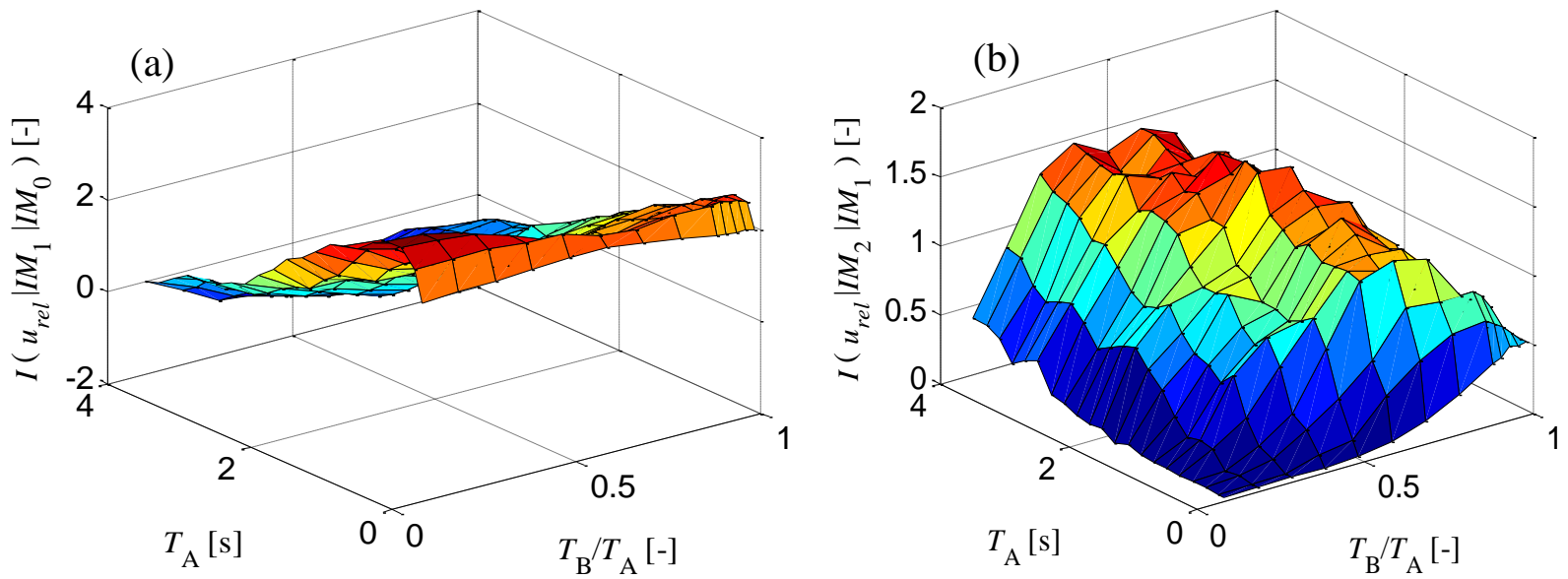

Figure 5. Variation with $T_{\mathrm{A}}$ and $T_{\mathrm{B}} / T_{\mathrm{A}}$ of the RSM for: (a) $I M_{1}$ with respect to $I M_{0}$, and (b) $I M_{2}$ with respect to $I M_{1}$.

It is noteworthy that Figure 2 through 5 are plotted using a discretization with intervals equal to 0.1 for $T_{\mathrm{B}} / T_{\mathrm{A}} \leq 0.9$ and to 0.025 for $T_{\mathrm{B}} / T_{\mathrm{A}}>0.9$. This discretization with different intervals was adopted to better describe the higher variability exhibited in the range $T_{\mathrm{B}} / T_{\mathrm{A}}>0.9$ by the quantities under study.

\section{PSDA RESULTS FOR ADJACENT BUILDINGS MODELED AS NOLINEAR HYSTERETIC MDOF SYSTEMS}




\subsection{Case study description}

In this section, PSDA is applied to evaluate the PSDM for the case study of two adjacent steel moment-resisting frame buildings with nonlinear hysteretic behavior. The same buildings already analyzed in [17] are considered here (Figure 6). Building A is an eight-story shear-type building with constant inter-story stiffness $k_{\mathrm{A}}=628,801 \mathrm{kN} / \mathrm{m}$ and floor mass $m_{\mathrm{A}}=454,550 \mathrm{~kg}$, while building $\mathrm{B}$ is a four-story shear-type building with constant inter-story stiffness $k_{\mathrm{B}}=$ $470,840 \mathrm{kN} / \mathrm{m}$ and floor mass $m_{\mathrm{B}}=454,550 \mathrm{~kg}$. The story heights are equal to $3.2 \mathrm{~m}$ for both buildings. A Rayleigh-type damping matrix is used to model the inherent viscous damping in the two systems. The matrix is built by assigning a damping ratio $\zeta_{\mathrm{R}}=2 \%$ to the first two vibration modes of each system considered independently from the other. The fundamental vibration periods of the two buildings are $T_{\mathrm{A}}=0.915 \mathrm{~s}$ and $T_{\mathrm{B}}=0.562 \mathrm{~s}$, respectively.

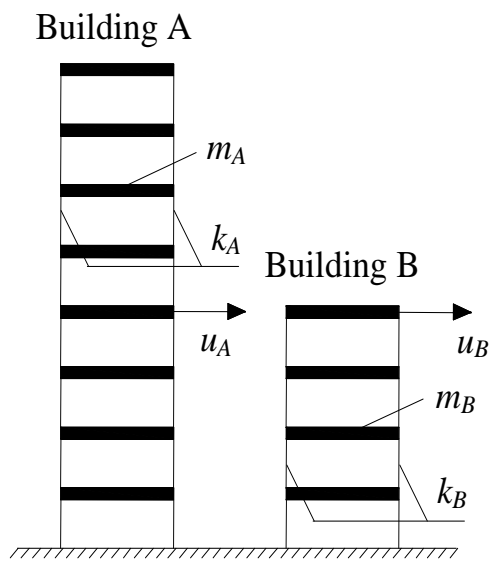

Figure 6. Models of buildings A and B.

A bilinear hysteretic constitutive model with kinematic hardening describes the relationship between the inelastic inter-story restoring force and inter-story drift [32]. This constitutive model for building $i$ (with $i=\mathrm{A}, \mathrm{B}$ ) is defined by the yield force, $F_{y, i}$, and by the ratio of the post-yield to initial stiffness, $r_{i}$, which is assumed equal to 0.05 for both models. The inter-story yield forces for system A and B are respectively $F_{y, \mathrm{~A}}=6871.4 \mathrm{kN}$ and $F_{y, \mathrm{~B}}=3755.4 \mathrm{kN}$ and are derived from [33]. The participation factors for the first vibration modes of the two buildings are: $\gamma_{A}=$ 0.855 and $\gamma_{B}=1.241$.

\subsection{Linear and bilinear PSDMs}

Cloud analysis is applied to this case study by employing the same set of 240 records already considered in the previous section. Since the buildings are expected to undergo significant inelastic deformations for a large number of records, the EDP samples corresponding to values of the peak inter-story drift (IDR) demand for the systems higher than $4 \%$ are discarded in developing the PSDM. The 4\% limit is taken from FEMA 356 [41] and corresponds to the collapse limit states for steel moment-resisting frame buildings. A reduced set of samples (consisting of 234 out of 240 relative displacement responses conditioned on not exceeding the IDR limit of 4\%) is used to derive the PSDMs conditioned on no collapse for this application example. The information on structural collapse can be incorporated in the model by estimating the supplementary probability of collapse as a function of the seismic intensity, as described in [42]. 
Two sets of linear and bilinear PSDMs are developed for each of the four IMs considered in the previous section. It is found that $I M_{0}=P G A$ provides very high relative displacement demand dispersions (close to 0.45 for both linear and bilinear PSDM), whereas the results obtained using $I M_{2}$ and $I M_{3}$ are practically identical, given that the correlation coefficient $\rho_{\mathrm{BA}}$ assumes a very low value, i.e., 0.0064. Thus, only the results obtained for $I M_{1}$ and $I M_{2}$ are shown and commented hereinafter.

Figure 7(a) and (b) report the response samples and the fitted median demand obtained by using the linear and bilinear PSDMs for $I M_{1}$ and $I M_{2}$, respectively. For the linear PSDMs, the values of the regression parameter $b$ assume values contained between 0.6 and 0.7 .
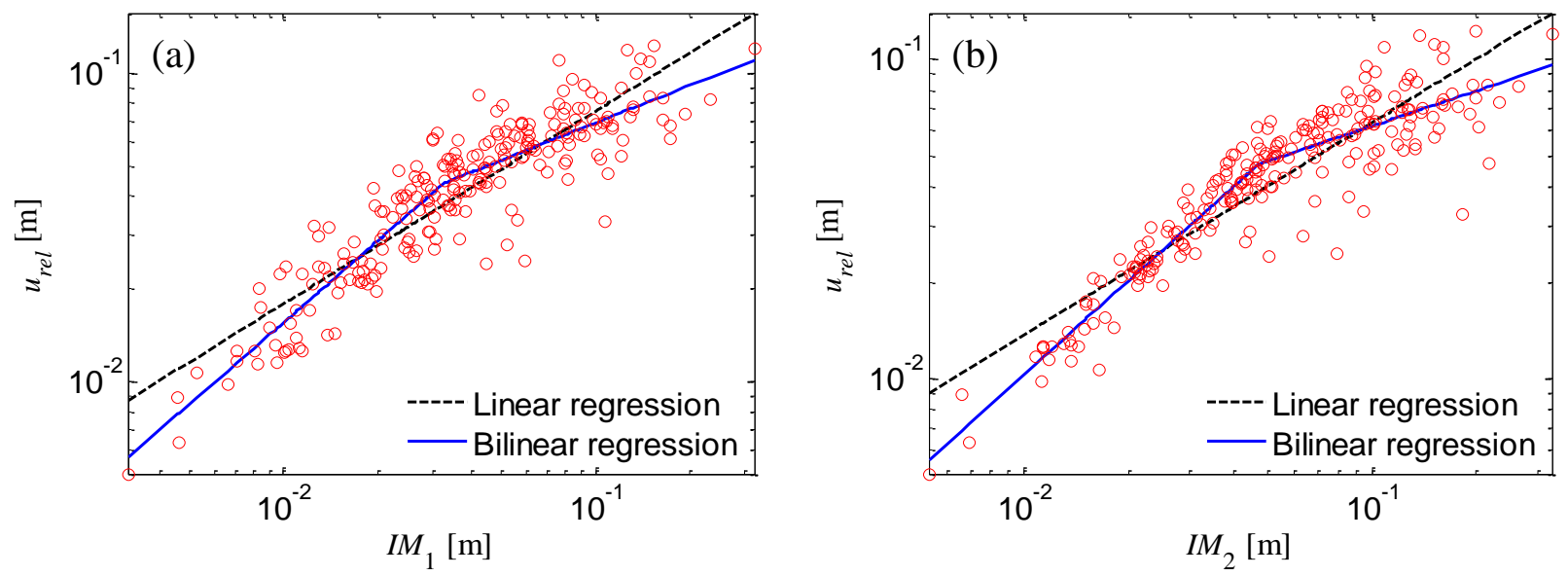

Figure 7. Comparison of linear and bilinear regression PSDMs in the log-log plane by using as $I M$ : (a) $I M_{1}$, and (b) $I M_{2}$.

Figure 8 plots the variation with $I M_{1}$ and $I M_{2}$ of the dispersion corresponding to the two (linear and bilinear) PSDM models. With regard to the linear PSDM, the observed relative displacement demand dispersions, measured by $\beta_{i}(i=1,2)$, are higher than those obtained for the SDOF linear systems. Furthermore, in this specific case study, the relative displacement demand dispersion corresponding to the use of $I M_{2}\left(\beta_{2}=0.250\right)$ is only slightly lower than the dispersion corresponding to the use of $I M_{1}\left(\beta_{1}=0.262\right)$.

The bilinear regression model gives better results in terms of efficiency than the linear model. In fact, in the case of $I M_{1}$, the dispersion corresponding to the bilinear regression model is equal to 0.231 and 0.236 respectively for 'low' and 'high' $I M$ values. In the case of $I M_{2}$, the dispersion corresponding to the bilinear PSDM model is equal to 0.146 and 0.248 respectively for 'low' and 'high' $I M$ values. These values of the dispersion confirm the superiority of $I M_{2}$ in terms of efficiency in the case of linear structural behavior, consistently with the results reported in the previous section for the SDOF linear models. As expected, in the case of nonlinear structural response, the efficiency of $I M_{2}$ tends to reduce. The bilinear model is superior to the linear model also considering a goodness-of-fit criterion based on the standard error of the regression, $S$ [43]. This parameter represents the average distance between the observed values and the regression line, so that lower values of it correspond to higher accuracy of fit. In the case of the linear model, $S$ coincides with the dispersion $\beta_{i}$ and it assumes the values $S=0.262$ for $I M=I M_{1}$ and $S$ $=0.250$ for $I M=I M_{2}$, whereas in the case of the nonlinear regression model it assumes the values $S=0.233$ for $I M=I M_{1}$ and $S=0.206$ for $I M=I M_{2}$. 

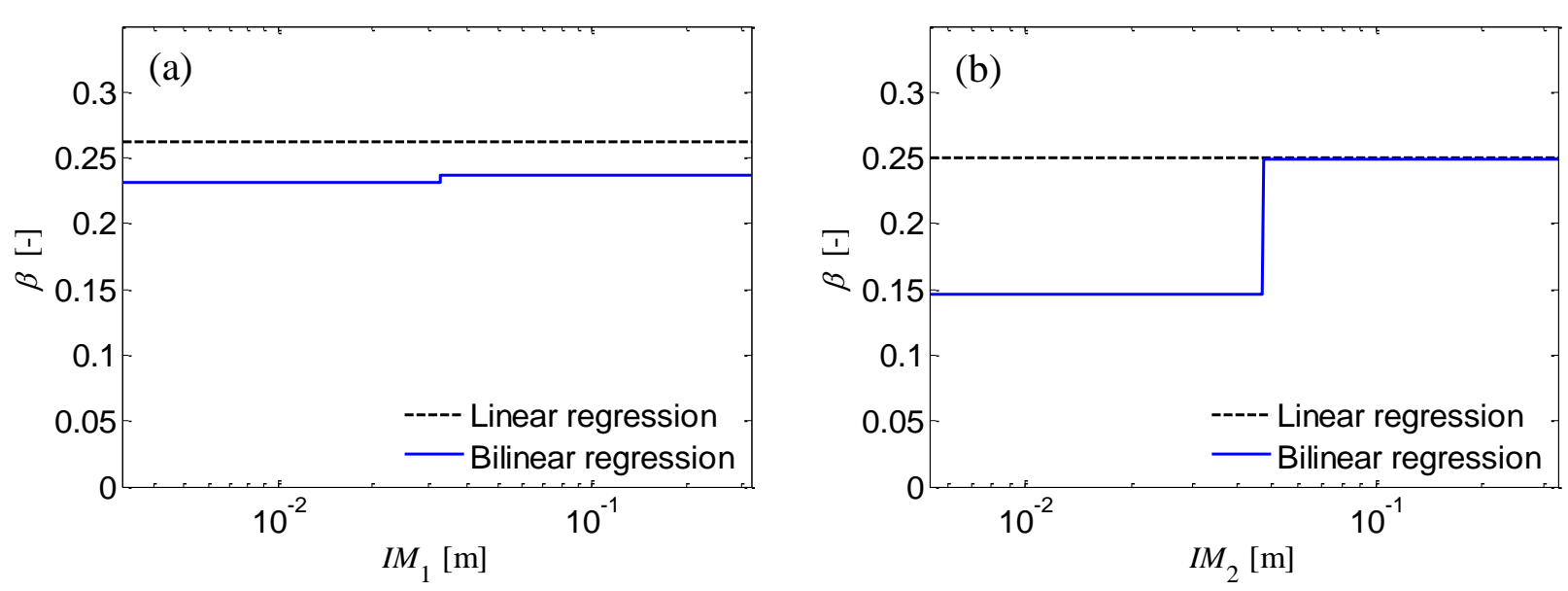

Figure 8. Dispersion of linear and bilinear regression models as a function of: (a) $I M_{1}$, and (b) $I M_{2}$.

The sufficiency of the $I M \mathrm{~s}$ with respect to $M$ and $R$ is evaluated by performing a linear regression of the residuals of both the linear and bilinear regression model $\ln \varepsilon_{k} \mid I M_{i}$ ( $k=1,2, . ., N_{g m}$ ) on $M$ and $R$, considered one at a time [29]. Figure 9(a) and (b) show the residuals, regression lines, and p-values corresponding to the linear regression model by using $I M_{1}$ and $I M_{2}$ for $M$ and $R$, respectively. Figure 9(c) and (d) show the same quantities as those reported in Figure 9(a) and (b), respectively, corresponding to the bilinear regression model. Based on a cut-off pvalue of 0.05 , the null hypotheses that the slopes of the regression lines are zero cannot be rejected for any of the $I M \mathrm{~s}$ considered and for both $M$ and $R$, with the exception of the case corresponding to the bilinear regression based on $I M_{1}$ for $M$.
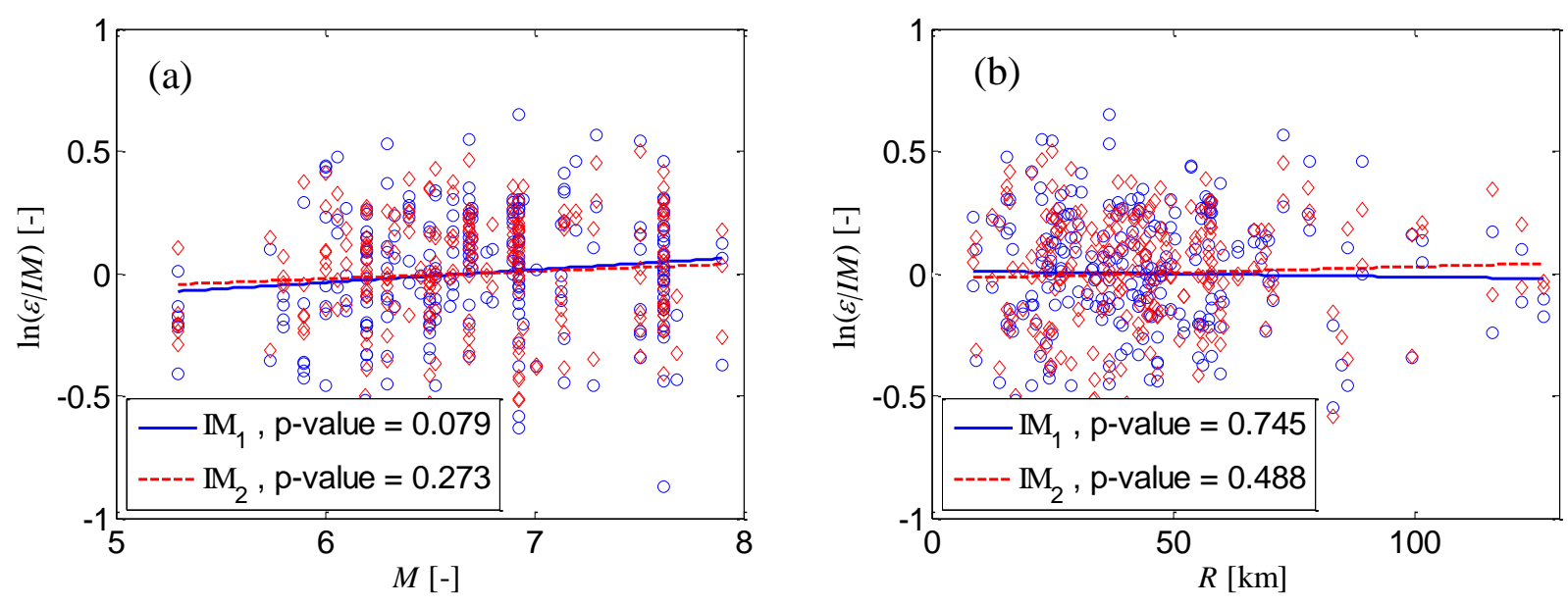

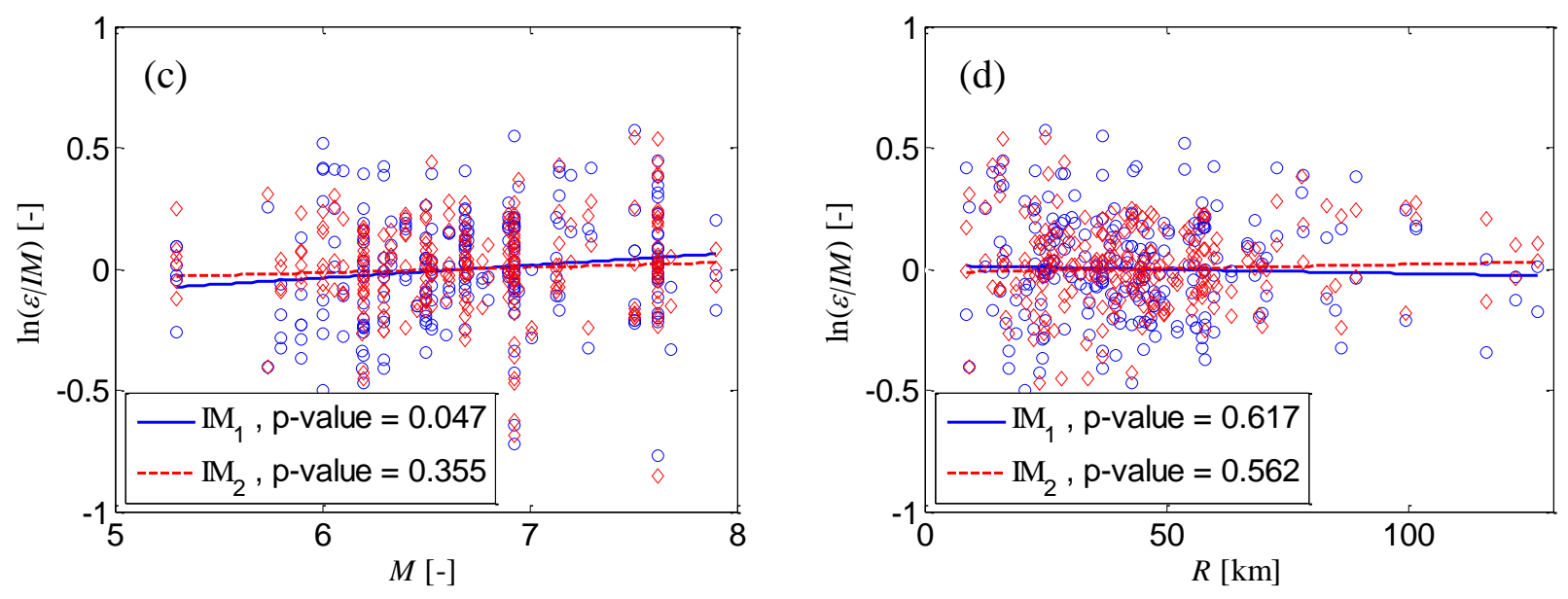

Figure 9. Cloud regression plots for: (a) linear regression model and $M$; (b) linear regression model and $R$; (c) bilinear regression model and $M$; and (d) bilinear regression model and $R$.

\subsection{Comparison of linear and bilinear PSDMs for seismic risk assessment}

In this section, the results of a seismic risk assessment analysis obtained using the linear and bilinear PSDMs developed in the previous section are compared. The fitted linear and bilinear PSDMs are employed to estimate via Eqn. (6) the probability of pounding conditioned on no collapse for different values of the separation distance $\xi$ in the range between $0 \mathrm{~m}$ and $0.2 \mathrm{~m}$ and for different values of the employed IMs. Figure 10 shows the fragility curves obtained using $I M_{2}$ for $\xi=0.05 \mathrm{~m}$ (Figure 10(a)) and $\xi=0.09 \mathrm{~m}$ (Figure 10(b)). It is noted that the fragility curve obtained for $\xi=0.05 \mathrm{~m}$ by employing the bilinear regression model exhibits a jump in correspondence of the breakpoint $I M^{*}$ between the two linear branches of the PSDM (from $P_{f}=$ 0.26 for values of $I M$ approaching $I M^{*}$ from below, to $P_{f}=0.36$ for values of $I M$ approaching $I M^{*}$ from above) due to the change of slope of the median response and of the dispersion, as also observed in [38]. For the lower value of the separation distance, the $I M_{2}$ value corresponding to a $50 \%$ probability of pounding is higher in the case of the bilinear regression model than in the case of the linear regression model. For the higher value of the separation distance, higher vulnerability is calculated by employing the linear rather than the bilinear model. It is noteworthy that, for $\xi=0.09 \mathrm{~m}$, the jump in the fragility curve obtained using the bilinear regression model is not visible, due to the fact that $P_{f}=0$ for $I M=I M^{*}$. Figure 10 also shows the numerical fragility curves obtained through incremental dynamic analysis (IDA) [27]. These curves are obtained by scaling all the 240 records to discrete common $I M$ values and directly comparing the response samples to the capacity (i.e., the separation distance). The numerical fragility curves obtained through cloud analysis by employing a bilinear PSDM are close to the corresponding curves estimated using IDA and are derived at only a small fraction of the computational cost of the corresponding IDA-based curves. However, it is worth noting that the IDA results, used here as reference solutions, cannot be considered "exact", since they are obtained by scaling, sometimes even by a significant amount, the seismic records. 

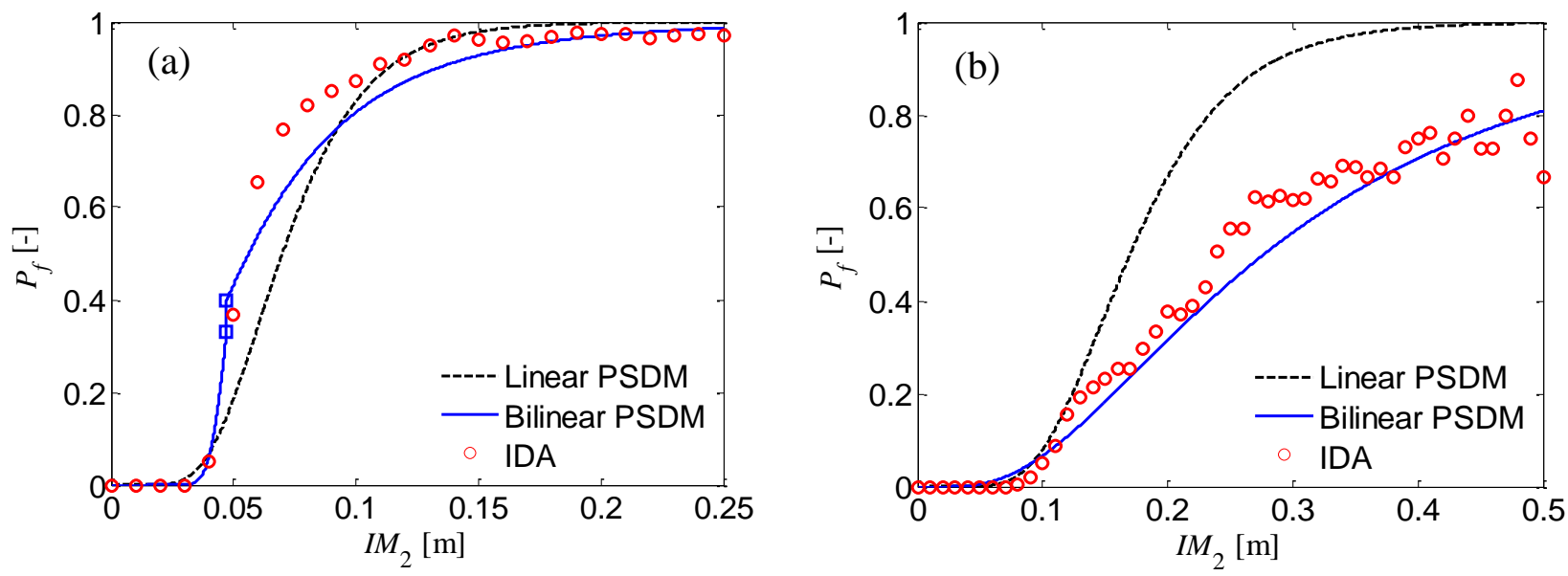

Figure 10. Fragility curves obtained by using linear PSDM, bilinear PSDM, and IDA for $I M_{2}$ and different values of the separation distance: (a) $\xi=0.05 \mathrm{~m}$ (square markers denote the jump of the fragility curve at $I M^{*}$ ), and (b) $\xi=$

$0.09 \mathrm{~m}$.

The MAF of pounding for a given deterministic separation distance $\xi, v_{f}=v_{E D P}(\xi)$, is also evaluated through the procedure reported in [16] by assuming that the buildings are located in Los Angeles, CA. The information on the seismic hazard curve for the site is taken from the United States Geological Survey's (USGS) website [44], which provides the MAF of exceedance for the $P G A$ and spectral accelerations at discrete periods in the range between $0.1 \mathrm{~s}$ and $4.0 \mathrm{~s}$. The MAFs of exceedance of the proposed $I M \mathrm{~s}, v_{I M}(\mathrm{im})$, are obtained by interpolating the available hazard curves. Figure 11(a) reports the hazard curve $v_{I M}(\mathrm{im})$ for $I M_{1}$ and $I M_{2}$, whereas Figure 11(b) shows the MAF of pounding for $\xi$ in the range between $0 \mathrm{~m}$ and $0.3 \mathrm{~m}$ obtained for $I M_{2}$ through the linear PSDM, bilinear PSDM, and IDA.
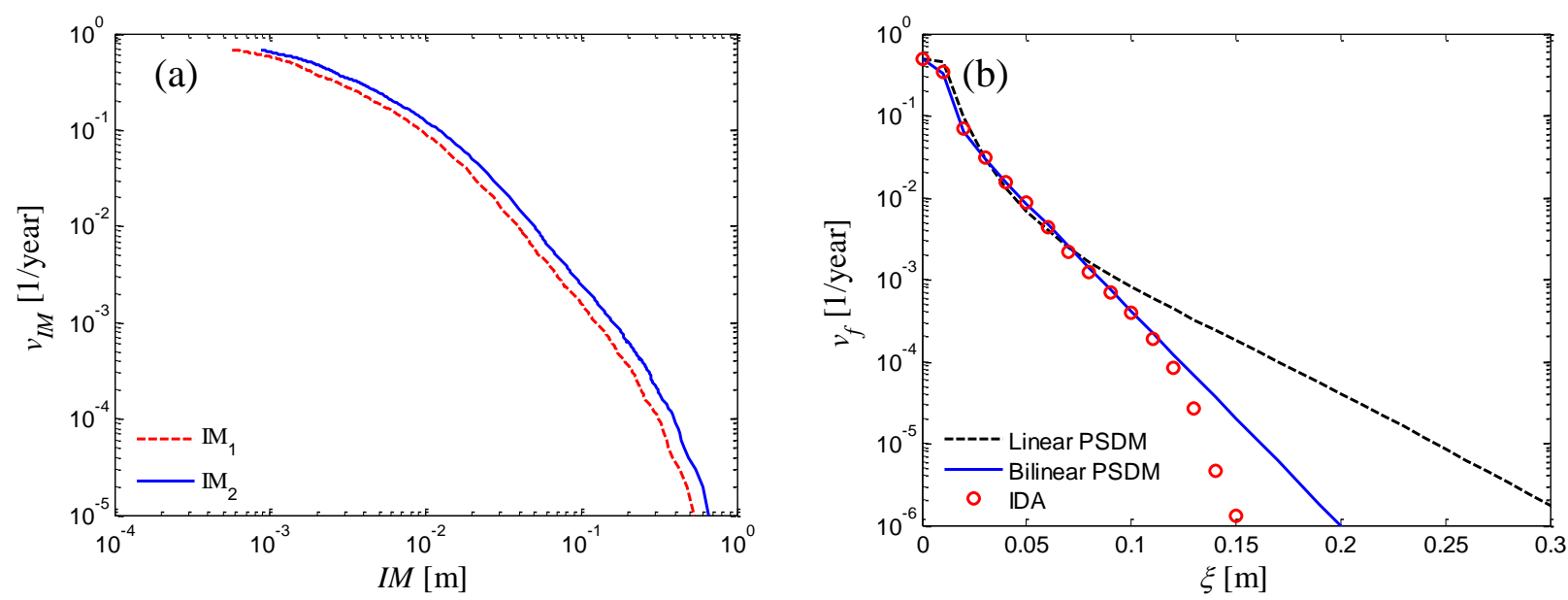

Figure 11. Seismic risk analysis: (a) hazard curve for $I M_{1}$ and $I M_{2}$ at the selected location (Los Angeles, CA), and (b) MAF of pounding based on $I M_{2}$ for different values of the separation distance and estimated using linear PSDM, bilinear PSDM, and IDA.

The three techniques provide very similar results for separation distances between $0 \mathrm{~m}$ and $0.07 \mathrm{~m}$. For $\xi$ values higher than $0.07 \mathrm{~m}$, the results obtained using cloud analysis in conjunction with the bilinear regression model are close to the results obtained through IDA, whereas the linear model provides highly conservative estimates of the pounding risk. Similar results are 
obtained by using $I M_{1}$ and, thus, are not reported here due to space constraints.

\section{CONCLUSIONS}

This paper proposes an efficient and accurate probabilistic seismic demand model (PSDM) for assessing the risk of pounding between adjacent buildings within modern Performance-Based Earthquake Engineering (PBEE) frameworks. The model is defined using different advanced intensity measures, based on well-known design rules for estimating the buildings' separation distance, and a bilinear regression model for the response samples obtained by cloud analysis.

An extensive parametric study is carried out for adjacent buildings modeled as single-degreeof-freedom linear systems under a suite of 240 natural ground motion records. In this case, the bilinear regression model reduces to a linear regression model. The parametric study results reveal that intensity measures $(I M)$ based on rules for separation distance design, such as the square root of the sum of the squares $\left(I M_{2}\right)$ and the double difference combination $\left(I M_{3}\right)$ rules, are superior in terms of efficiency to more common $I M$, i.e., to $I M_{0}=P G A$, and to the spectral displacement at the fundamental period of the taller building $\left(I M_{1}\right)$. It is also shown that (1) $I M_{2}$ and $I M_{3}$ are sufficient with respect to the magnitude $(M)$ and source-to-site distance $(R)$ for a wide range of system properties, (2) $I M_{2}$ and $I M_{3}$ are always more sufficient than $I M_{0}=P G A$ and $I M_{1}$, and (3) $I M_{2}$ and $I M_{3}$ give similar results in terms of both efficiency and sufficiency, even though $I M_{3}$ provides less biased estimates of the peak relative displacement than $I M_{2}$.

A case study of two realistic steel buildings modeled as nonlinear hysteretic multi-degree-offreedom shear-type systems is also analyzed in detail. Linear and bilinear PSDMs are considered to describe the relative displacement demand at the most likely pounding location. Based on the results of the study, the following conclusions are drawn: (1) $I M_{2}$ and $I M_{3}$ are more efficient intensity measures than $P G A$ and $I M_{1}$, even when inelastic seismic behavior is taken into account; (2) the $I M$ efficiency is higher while using the bilinear PSDM than the linear PSDM, at least in the linear behavior range; (3) the bilinear PSDM provides a more accurate description of the seismic demand than the linear PSDM, since it is able to account for the changes of the relative displacement demand (in terms of median value and dispersion) due to structural yielding; and (4) the use of a bilinear PSDM in conjunction with cloud analysis provides seismic pounding risk estimates that are very close to those obtained through incremental dynamic analysis at a small fraction of the computational cost and without scaling the records. Thus, the bilinear PSDM in conjunction with cloud analysis is recommended for seismic pounding risk analysis of buildings with nonlinear structural behavior.

It is noted here that, while the scope of the proposed PSDM is limited to the evaluation of the pounding probability, further studies should be carried out to assess its adequacy for evaluating the performance of adjacent buildings subject to seismic pounding hazard. These studies could use mechanical models already available in the literature to simulate the effects of pounding between adjacent buildings, and could extend the probabilistic analyses (which focus only on relative displacement response assessment in the present study) to account for the variability of damage and losses of the building components.

\section{ACKNOWLEDGEMENTS}

The third author gratefully acknowledges partial support of this research by the Louisiana Department of Wildlife and Fisheries through award \#724534. Any opinions, findings, conclusions, or recommendations expressed in this publication are those of the authors and do not necessarily reflect the views of the sponsors. 


\section{REFERENCES}

1. Anagnostopoulos SA. Pounding of buildings in series during earthquakes. Earthquake Engineering \& Structural Dynamics 1988; 16 (3): 443-456.

2. Cole GL, Dhakal RP, Turner FM. Building pounding damage observed in the 2011 Christchurch earthquake. Earthquake Engineering \& Structural Dynamics 2012; 41 (5):893913.

3. Filiatrault A., Wagner P. and Cherry S. Analytical prediction of experimental building pounding. Earthquake Engineering \& Structural Dynamics 1995; 24 (8):1131-1154.

4. Papadrakakis M, Mouzakis HP. Earthquake simulator testing of pounding between adjacent buildings. Earthquake Engineering \& Structural Dynamics 1995; 24 (6):811-834.

5. Polycarpou PC, Komodromos P. Earthquake-induced poundings of a seismically isolated building with adjacent structures. Engineering Structures 2010; 32 (7):1937-1951.

6. Jankowski R. Non-linear viscoelastic modelling of earthquake-induced structural pounding. Earthquake Engineering \& Structural Dynamics 2005; 34 (6):595-611.

7. Efraimiadou S, Hatzigeorgiou GD, Beskos DE. Structural pounding between adjacent buildings subjected to strong ground motions. Part I: The effect of different structures arrangement. Earthquake Engineering \& Structural Dynamics 2013; 42 (10):1509-1528.

8. Skrekas P, Sextos A, Giaralis A. Influence of bi-directional seismic pounding on the inelastic demand distribution of three adjacent multi-storey R/C buildings. Earthquakes and Structures 2014; 6 (1):71-87.

9. Takabatake H, Yasui M, Nakagawa Y, Kishida A. Relaxation method for pounding action between adjacent buildings at expansion joint. Earthquake Engineering \& Structural Dynamics 2014; 43 (9):1381-1400.

10. Tubaldi E. Dynamic behavior of adjacent buildings connected by linear viscous/viscoelastic dampers. Structural Control Health Monitoring 2015; 22 (8):1086-1102.

11. Raheem SA. Mitigation measures for earthquake induced pounding effects on seismic performance of adjacent buildings. Bulletin of Earthquake Engineering 2014; 4 (12):17051724.

12. Building Center of Japan (BCJ). Structural provisions for building structures: Tokyo, Japan 1997.

13. International conference of building officials (ICBO). Uniform building code (UBC) 1997; Whittier, CA, USA.

14. Construction and Planning Administration Ministry of Interior. Seismic Provisions. Building Code (TBC): Taipei, Taiwan 1997.

15. European Committee for Standardization (ECS). Eurocode 8 - Design of structures for earthquake resistance (EN1998) 2005; Brussels, Belgium.

16. Tubaldi E, Barbato M, Ghazizadeh SA. Probabilistic performance-based risk assessment approach for seismic pounding with efficient application to linear systems. Structural Safety 2012; 36-37: 14-22.

17. Barbato M, Tubaldi E. A probabilistic performance-based approach for mitigating the seismic pounding risk between adjacent buildings. Earthquake Engineering \& Structural Dynamics 2013; 42 (8): 1203-1219.

18. Chase JG, Boyer F, Rodgers GW, Labrosse G, MacRae GA. Probabilistic risk analysis of structural impact in seismic events for linear and nonlinear systems. Earthquake Engineering \& Structural Dynamics 2014; 43 (10), pages 1565-1580.

19. Aslani H, Miranda E. Probability-based seismic response analysis. Engineering Structures 2005; 27 (8), 1151-1163. 
20. Padgett JE, Nielson BG, DesRoches R. Selection of optimal intensity measures in probabilistic seismic demand models of highway bridge portfolios. Earthquake Engineering \& Structural Dynamics 2008; 37 (5): 711-725.

21. Rajeev P, Franchin P and Tesfamariam S (2014) "Probabilistic Seismic Demand Model for RC Frame Buildings Using Cloud Analysis and Incremental Dynamic Analysis", Proc. of 10th National Conference on Earthquake Engineering, Anchorage, Alaska.

22. Mackie KR, Stojadinovic B. Comparison of incremental dynamic, cloud, and stripe methods for computing probabilistic seismic demand models. Proceedings of the 2005 ASCE Structures Congress 2005; New York, NY.

23. Porter KA. An overview of PEER's performance-based earthquake engineering methodology. Proceedings of the $9^{\text {th }}$ International Conference on Application of Statistics and Probability in Civil Engineering (ICASP9) 2003; San Francisco, California; 973-980.

24. Zhang Y, Acero G, Conte J, Yang Z, Elgamal A. Seismic reliability assessment of a bridge ground system. Proceedings of the $13^{\text {th }}$ World Conference on Earthquake Engineering 2004; Vancouver, Canada.

25. Cornell CA, Jalayer F, Hamburger RO, Foutch DA. Probabilistic Basis for 2000 SAC Federal Emergency Management Agency Steel Moment Frame Guidelines, Journal of Structural Engineering 2002; 128 (4), 526-533.

26. Luco N, Cornell CA. Structure-specific scalar intensity measures for near-source and ordinary earthquake ground motions. Earthquake Spectra 2007; 23 (2):357-92.

27. Vamvatsikos D, Cornell CA. Incremental dynamic analysis. Earthquake Engineering \& Structural Dynamics 2002; 31 (3):491-514.

28. Shome N, Cornell CA. Probabilistic seismic demand analysis of nonlinear structures. Reliability of Marine Structures Program 1999; Report No. RMS-35. Department of Civil and Environmental Engineering, Stanford University, California.

29. Vega J, Del Rey I, Alarcon E. Pounding force assessment in performance-based design of bridges. Earthquake Engineering \& Structural Dynamics 2009; 38 (13): 1525-1544.

30. Ebrahimian H, Jalayer F, Lucchini A, Mollaioli F, Manfredi G. Preliminary ranking of alternative scalar and vector intensity measures of ground shaking. Bull. Earthquake Eng. 2015.

31. Lopez-Garcia D, Soong TT. Assessment of the separation necessary to prevent seismic pounding between linear structural systems. Probabilistic Engineering Mechanics 2009; 24(2): 210-223.

32. Lopez-Garcia D, Soong TT. Evaluation of current criteria in predicting the separation necessary to prevent seismic pounding between nonlinear hysteretic structural systems. Engineering Structures 2009; 31 (5): 1217-1229.

33. Lin JH. Evaluation of seismic pounding risk of buildings in Taiwan. Chinese Institute of Engineers 2005; 28 (5): 867-72.

34. Mackie KR, Stojadinovic B. Fragility Curves for Reinforced Concrete Highway Overpass Bridges. $13^{\text {th }}$ World Conference on Earthquake Engineering 2004; Vancouver, BC.

35. Baker JW. Probabilistic structural response assessment using vector-valued intensity measures. Earthquake Engineering \& Structural Dynamics 2007; 36 (13): 1861-1883.

36. Kasai K, Jagiasi RA, Jeng V. Inelastic vibration phase theory for seismic pounding. Journal of Structural Engineering 1996; 122 (10): 1136-1146.

37. Tubaldi E, Barbato M. DISCUSSION: Probabilistic risk analysis of structural impact in seismic events for linear and nonlinear systems. Earthquake Engineering \& Structural Dynamics 2015; 44 (3), 491-493.

38. Bai JW, Gardoni P, Hueste MBD. Story-specific demand models and seismic fragility 
estimates for multi-story buildings. Structural Safety 2011; 33(1), 96-107.

39. Barenblatt GI. Dimensional Analysis, Gordon and Breach Science Publishers: New York 1987.

40. Baker JW, Jayaram N, Shahi S. Ground Motion Studies for Transportation Systems. PEER Website 2011.

41. Federal Emergency Management Agency. FEMA 356: Prestandard and Commentary for the Seismic Rehabilitation of Buildings. Washington DC, 2000.

42. Shome N, Cornell CA. Structural seismic demand analysis: consideration of 'Collapse'. $8^{\text {th }}$ ASCE Specialty Conference on Probabilistic Mechanics and Structural Reliability 2007; University of Notre Dame, South Bend, IN.

43. Ott RL, Longnecker M. An introduction to Statistical Methods and Data Analysis (6 ${ }^{\text {th }}$ edn). Brooks/Cole, Cengage Learning, Belmont, CA.

44. USGS, United States Geological Survey. (http:// www.usgs.gov/). 